\title{
Analysis of superconducting thin films in a modern FIB/SEM dual-beam instrument
}

Lukas Grünewald $^{1}$, Daniel Nerz ${ }^{1}$, Marco Langer ${ }^{2}$, Sven Meyer ${ }^{2}$, Nico Beisig ${ }^{2}$, Pablo Cayado ${ }^{2}$, Ruslan Popov $^{2}$, Jens Hänisch ${ }^{2}$, Bernhard Holzapfel ${ }^{2}$ and Dagmar Gerthsen ${ }^{3}$

${ }^{1}$ Karlsruhe Institute of Technology (KIT), Laboratory for Electron Microscopy (LEM), Engesserstraße 7, 76131 Karlsruhe, Germany, United States, ${ }^{2}$ Karlsruhe Institute of Technology (KIT), Institute for Technical Physics (ITEP), Hermann-von-Helmholtz-Platz 1, 76344 Eggenstein-Leopoldshafen, Germany, United States, ${ }^{3}$ Laboratorium für Elektronenmikroskopie, Karlsruher Institut für Technologie (KIT), Engesserstr. 7, 76131 Karlsruhe, Germany, United States

Thin-film technology is used for the synthesis of many application-relevant materials, e.g. for the fabrication of superconducting thin films for high-power applications [1]. The superconducting properties of such a thin film are strongly dependent on the microstructure because structural defects can act as fluxpinning centers if they have favorable size, shape, and distribution within the superconducting matrix [2]. This increases the critical current density, which is important for technical applications. (Scanning) transmission electron microscopy $((\mathrm{S}) \mathrm{TEM})$ in a dedicated $(\mathrm{S}) \mathrm{TEM}$ instrument is a powerful analysis tool for film characterization on the sub-nm (and sub- $\AA$ ) scale. However, TEM sample preparation and consecutive (S)TEM measurement in different instruments limit high-throughput analysis. Modern combined focused-ion-beam/scanning electron microscope (FIB/SEM) instruments are routinely used for TEM sample preparation and SEM imaging and can be additionally equipped with STEM- (for STEMin-SEM imaging) and energy-dispersive x-ray spectroscopy- (EDXS) detectors, resulting in a large versatility for material analysis [3].

In this work, we demonstrate how correlative SEM, EDXS, and STEM-in-SEM in a Thermo Fisher Helios G4 FX FIB/SEM dual-beam instrument, equipped with a dedicated STEM holder and a Bruker XFlash 6|60 EDXS system, can be used for sample preparation and structural and chemical characterization within one instrument [3]. The investigated thin films consist of superconducting $\mathrm{Ba}\left(\mathrm{Fe}_{0.92} \mathrm{Co}_{0.08}\right)_{2} \mathrm{As} 2(\mathrm{Ba} 122)$ and rare-earth barium copper oxide $\mathrm{REBa}_{2} \mathrm{Cu}_{3} \mathrm{O}_{7-\delta}(\mathrm{REBCO}$, with $\mathrm{RE}=\mathrm{Y}, \mathrm{Gd}, \mathrm{Er}, \ldots)$ and are grown by pulsed laser and chemical solution deposition on different substrates.

Fig. 1 shows the SEM-EDXS analysis of a thin $\mathrm{Ba} 122$ film grown on $\mathrm{CaF}_{2}$ that contains precipitates with sizes of about $80 \mathrm{~nm}$ (bright spots in Fig. 1(a)). EDXS mapping was performed in the region marked in Fig. 1(a) for chemical analysis of the film and the precipitates. Due to the small thickness $(60 \mathrm{~nm})$ of the Ba122 film, a low electron energy of $2 \mathrm{keV}$ was used to maximize the spatial resolution while still exciting all necessary x-ray lines, i.e. the Ba M-line-, Fe L-line-, Co L-line, and As L-line-families. Due to the low $\mathrm{x}$-ray yield at $2 \mathrm{keV}$, principal component analysis (PCA) is applied to increase the signal-to-noise ratio (Fig. 1(b)) by dimensionality reduction of the dataset [4]. The precipitates show a high Fe/Co content in the qualitative net-intensity elemental maps (Fig. 1(c)) in agreement with TEM results [5]. STEM-in-SEM imaging of Ba122 shows a reduced intensity in high-angle annular dark-field (HAADF) Z-contrast at planar defects (dotted arrows, Fig. 1(e)). Their dark appearance in HAADF-STEM images is similar to STEM images taken in a dedicated (S)TEM [5], where $O$ was detected at these defects. By in-situ preparation and STEM-in-SEM imaging, it was possible to eliminate possible oxidation of these planar defects during sample transport between the FIB/SEM and the dedicated (S)TEM instrument. Ba122(002) lattice fringes are visible in the simultaneously acquired bright-field (BF) image (Fig. 1(d)), which shows that BF STEM-in-SEM imaging can resolve sub-nm crystalline features. 
Examples for STEM-in-SEM imaging at $30 \mathrm{keV}$ of in-situ FIB-prepared samples from a GdBCO film with a thickness of $350 \mathrm{~nm}$ on a $\mathrm{MgO}$ substrate are shown in Fig. 2. Diffraction-contrast STEM-in-SEM dark-field imaging shows vertical, out-of-plane defects in the film (Fig. 2(a)). These defects correspond to threading dislocations, antiphase boundaries, and small-angle [001]-twist grain boundaries. For a better overview of the microstructure, additional plan-view samples were prepared by FIB milling and imaged by STEM-in-SEM (Fig. 2(b,c)). HAADF-STEM images of the same GdBCO film as in (a) show roundish precipitates, twin boundaries oriented along [110]-type directions (red arrows, as in [6]), and threading dislocations (orange arrows).

In conclusion, modern FIB/SEM instruments with STEM and EDXS detectors combine sample preparation with structural and chemical analysis down to the nm-scale and facilitates efficient thin-film analysis in a single instrument. This is particularly favorable for the investigation of materials that are susceptible to exposure to ambient air.

(a)

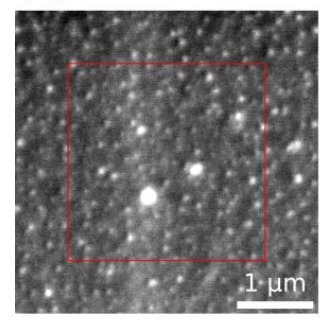

(b)

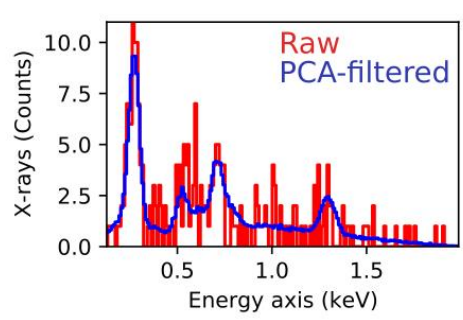

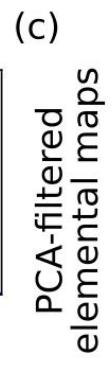
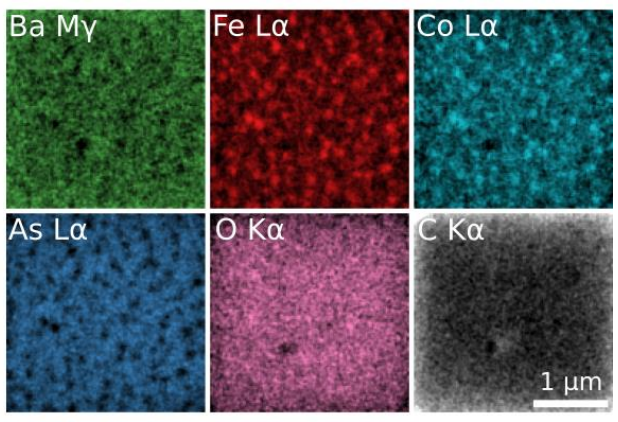
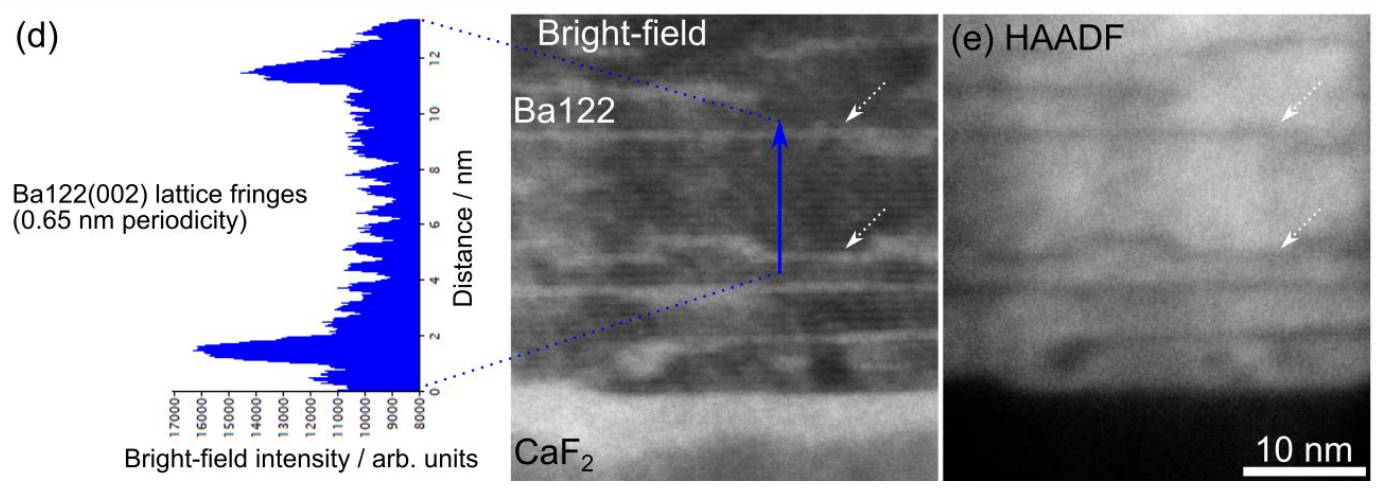

Figure 1. Low-energy SEM-EDXS analysis of a $\sim 60 \mathrm{~nm}$ thin Ba122 film on $\mathrm{CaF}_{2}$. (a) Overview secondary-electron SEM image with EDXS-mapped region marked by a square. (b) Example for raw (red) and PCA-filtered (blue) EDXS spectra from a single pixel of the EDXS dataset. The enhanced signal-tonoise ratio enables improved separation of the partially overlapping $\mathrm{x}$-ray lines. (c) Extracted net intensities of the x-ray lines for different elements. The precipitates are rich in Fe and Co. (d, e) Simultaneously acquired $30 \mathrm{keV}$ STEM-in-SEM (d) BF- and (e) HAADF-STEM cross-section images of a Ba122 film. The dotted arrows mark planar defects with increased/reduced intensity in the BF- and HAADF-STEM images. Ba122 lattice fringes are visible in the BF image as visible in the intensity line profile along the blue arrow. 

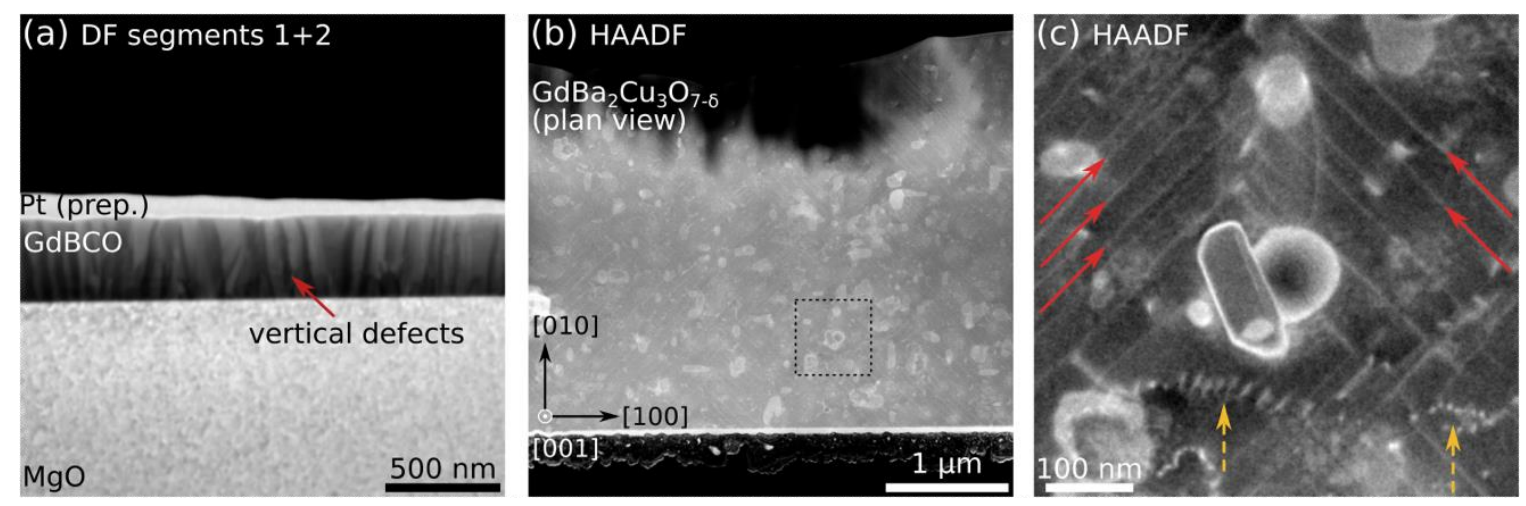

Figure 2. (a) STEM-in-SEM dark-field cross-section image of a $\sim 350 \mathrm{~nm}$ thin GdBCO film. The vertical defects show strong diffraction contrast. (b, c) Plan-view HAADF-STEM-in-SEM images of an in-situ prepared plan-view lamella of the GdBCO film. (c) Higher-magnification image of the region marked in (b). Twin boundaries (red arrows) and threading dislocations (yellow, dashed arrows) are visible besides precipitates.

\section{References}

[1] A.K. Jha, K. Matsumoto, Front. Phys. 7 (2019) 82.

[2] J.P.F. Feighan et al., Supercond. Sci. Technol. 30 (2017) 123001.

[3] C. Sun et al., J. Mater. Sci. 55 (2020) 13824-13835.

[4] P. Potapov, Ultramicroscopy 160 (2016) 197-212.

[5] L. Grünewald et al., Supercond. Sci. Technol. 34 (2021) 035005.

[6] R. Guzman et al., Phys. Rev. Mater. 1 (2017) 24801. 